\title{
Retracted: Anti-ribosomal-phosphoprotein autoantibodies penetrate to neuronal cells via neuronal growth associated protein, affecting neuronal cells in vitro
}

\author{
Shaye Kivity ${ }^{1}$, Yehuda Shoenfeld ${ }^{1}$, Maria-Teresa Arango ${ }^{1,2}$, Dolores J. Cahill ${ }^{3}$, \\ Sara Louise O'Kane ${ }^{3}$, Margalit Zusev' ${ }^{1}$, Inna Slutsky ${ }^{4}$, Michal Harel-Meir ${ }^{1}$, \\ Joab Chapman ${ }^{5}$, Torsten Matthias ${ }^{6}$ and Miri Blank ${ }^{1}$
}

Rheumatology, 2016, doi:10.1093/rheumatology/kew027

The above article from Rheumatology 'Anti-ribosomalphosphoprotein autoantibodies penetrate to neuronal cells via neuronal growth associated protein, affecting neuronal cells in vitro' by Shaye Kivity, Yehuda Shoenfeld, Maria-Teresa Arango, Dolores J. Cahill, Sara Louise O'Kane, Margalit Zusev, Inna Slutsky, Michal
Harel-Meir, Joab Chapman, Torsten Matthias and Miri Blank, published online on 06 May 2016 by Oxford University Press, has been retracted by agreement between the journal Editor, Dr Jacob M. van Laar, The British Society for Rheumatology and Oxford University Press. The retraction has been agreed due to the discovery of significant errors relating to methods and presentation of results.

\footnotetext{
${ }^{1}$ The Zabludowicz Center for Autoimmune Diseases, affiliated to Sackler Faculty of Medicine, Tel-Aviv University, Israel, ${ }^{2}$ Doctoral Program in Biomedical Sciences, Universidad del Rosario, Bogota, Colombia, ${ }^{3}$ School of Medicine and Medical Sciences, Conway Institute of Biomedical and Biomolecular Research, University College Dublin, Belfield, Ireland, ${ }^{4}$ Department of Physiology and Pharmacology, Sackler Faculty of Medicine, Tel Aviv University, Tel Aviv, ${ }^{5}$ Department of Neurology, Sagol Neuroscience Center, Sheba Medical Center, Tel-Hashomer, Israel and ${ }^{6}$ AESKU.KIPP Institute, Mikroforum Ring, Wendelsheim, Germany

Correspondence to: Yehuda Shoenfeld, Head of Zabludowicz Center for Autoimmune Diseases, Sheba Medical Center, Tel-Hashomer 52621, Israel.

E-mail: shoenfel@post.tau.ac.il
} 